\title{
Empfehlung der Schweizerischen Gesellschaft für Notfall- und Rettungsmedizin zur Triage in Schweizer Notfallstationen*
}

\author{
Wie weltweit alle Vereinigungen für Notfallmedizin empfiehlt auch die Schweize- \\ rische Gesellschaft für Notfall- und Rettungsmedizin (SGNOR) den Einsatz eines Tria- \\ gesystems in allen Notfallstationen, die zu irgendeinem Zeitpunkt mit dem Über- \\ schreiten ihrer Kapazitäten konfrontiert sind. Unter den Triagesystemen, welche die \\ höchste Zuverlässigkeit (Validität und Rehabilität) aufweisen, empfiehlt sie den Ge- \\ brauch des «Emergency Severity Index» (ESI) oder der «Echelle Suisse de Tri» (EST).
}

Olivier T. Rutschmann ${ }^{a}$, Robert S. Sieber ${ }^{\text {, }}$

Olivier W. Huglic

a PD, Service des Urgences, Département de Médecine Communautaire et de Premier recours, Hôpitaux Universitaires de Genève et Faculté de Médecine de Genève

b Ospedale Regionale di Lugano

c PD, Service des Urgences, Centre Hospitalier Universitaire Vaudois, Lausanne

\footnotetext{
* Dokument der Wissenschaftskommission der SGNOR (Vorsitz Prof. Roland Bingisser)
}

Übersetzung.

Dr. Beat Lehmann

Korrespondenz:

Olivier T. Rutschmann, PD Service des Urgences

Département de Médecine

Communautaire et

de Premier recours

Hôpitaux Universitaires de Genève et Faculté de Médecine de Genève

CH-1211 Genève 14

Tel. 0223728131

Fax 0223728141

olivier.rutschmann@sgnor.ch

\section{Triage in Notfallstationen}

In Anbetracht der chronischen Überlastung von Notfallstationen und der Unmöglichkeit, alle Patienten unmittelbar und gleichzeitig zu behandeln, ist der Triageprozess heute eine Realität und eine klare Forderung in allen Ländern mit einer strukturierten Notfallmedizin [1, 2, 4, 5]. Diese erste Phase der Patientenbetreuung in der Notfallstation wird gewöhnlich durch eine speziell ausgebildete Notfall-Pflegefachperson übernommen

Ziel der Triage ist es, die Patienten aufgrund ihrer Beschwerden und entsprechend der Dringlichkeit ihres Problems zeitgerecht der geeigneten Behandlung zuzuführen - dies unter Berücksichtigung von Infrastruktur und vorhandenen Ressourcen.

In den angelsächsischen Ländern, die seit Jahrzehnten über eine staatlich anerkannte klinische Notfallmedizin verfügen, wurden Richtlinien zum Ge- brauch von validierten Triagesystemen erarbeitet. So werden z.B. in Grossbritannien in allen Notfallstationen die National Triage Scale (früher: Manchester Triage Scale) angewendet [4], während in Australien die Australasian Triage Scale (ATS) [1] und in Kanada die Canadien Triage and Acuity Scale (CTAS) [5] gebraucht wird.

In den Vereinigten Staaten werden hingegen mehrheitlich Triagesysteme mit 5 Dringlichkeitsstufen verwendet, wobei die Notfallstation die Wahl hat zwischen dem Emergency Severity Index (ESI) und der CTAS [2].

\section{Triage in der Schweiz}

Kürzlich wurde eine Befragung unter 148 Schweizer Notfallstationen durchgeführt [8]. Von den 105 Notfallstationen, die den Fragebogen beantwortet haben, betreiben nur 55 (52\%) einen strukturierten Triage-
Eine Triage soll gewährleisten, dass Notfall-Patienten entsprechend ihrer Beschwerden und der Dringlichkeit zeitgerecht der geeigneten Behandlung zugeführt werden.

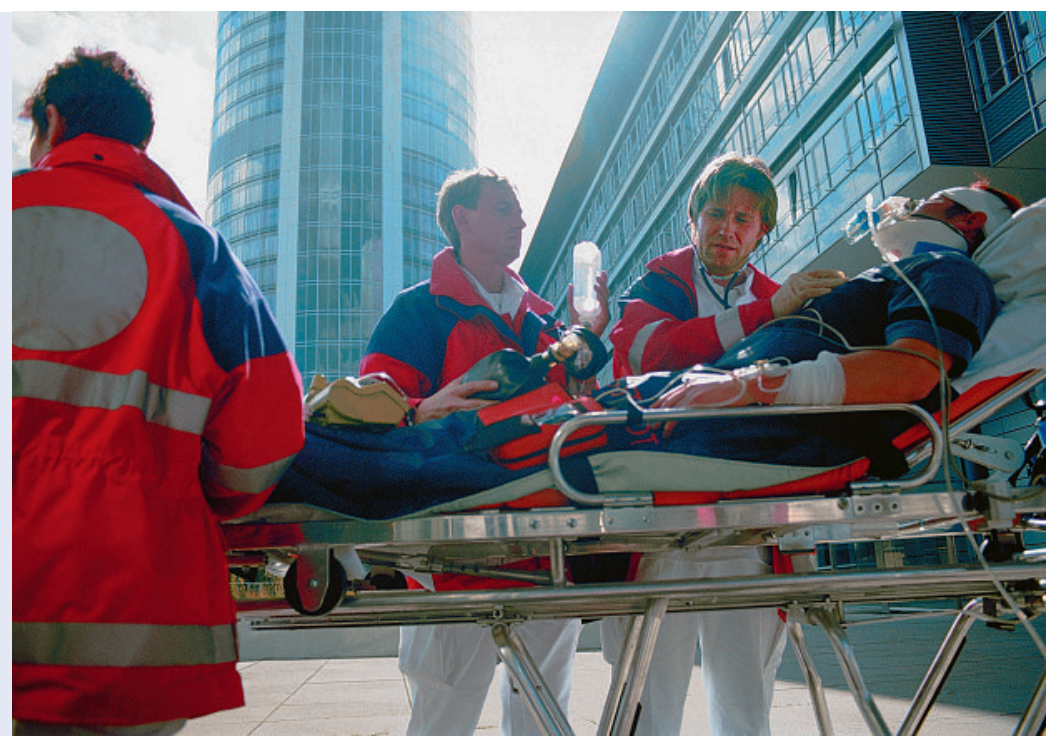


prozess. In den Notfallstationen mit weniger als 20000 Patienten pro Jahr $(\mathrm{n}=88)$ gebrauchten nur $48 \%$ ein Triagesystem, während $76 \%$ der grösseren Notfallstationen ( $\mathrm{n}=17 \mathrm{mit} \geq 20000$ Patienten) über ein solches System verfügten. Es besteht bisher keine Einigkeit zum Gebrauch der verschiedenen Triagesysteme. Die grosse Mehrheit der Notfallstationen $(\mathrm{n}=34 ; 62 \%)$ benutzt nicht validierte Triagesysteme mit nur drei Dringlichkeitsstufen.

Validierte Triagesysteme, die in der Schweiz gebraucht werden, sind das australische System (ATS) [1], das kanadische Protokoll (CTAS) [5], der Emergency Severity Index (ESI) aus den USA [2], das Genfer Triageprotokoll $($ Geneva Emergency Triage Scale $=$ GETS $)$ $[6,7]$, das kürzlich in Echelle Suisse de Tri (EST) umbenannt worden ist, und das Lausanner Instrument Echelle lausannoise de tri et de gravité (ELTG) [3]

\section{Empfehlung der Wissenschaftskommission der Schweizerischen Gesellschaft für Notfall- medizin und Rettungsmedizin (SGNOR)}

Mit der Einführung des Fähigkeitsausweises für klinische Notfallmedizin durch die FMH hat die SGNOR ein Curriculum zur Weiterbildung der zukünftigen Notfallmediziner und Qualitätsstandards für Schweizer Notfallstationen erarbeitet. Die SGNOR hat aufgrund der Heterogenität der Triagesysteme und vieler Nachfragen die folgenden von ihrer Wissenschaftskommission erarbeiteten Empfehlungen zum Gebrauch von Triagesystemen beschlossen:

- Alle Notfallstationen, die zu irgendeinem Zeitpunkt mit einem Überschreiten ihrer Kapazitäten konfrontiert sind, sollten über ein Triagesystem verfügen.

- Dieses Triagesystem muss nachgewiesenermassen valide und reliabel sein (publiziert in einem Peerreviewed Journal) und zur Optimierung der Ausgeglichenheit der Triage beitragen, indem Unterund Übertriage minimiert werden.

- Die Zahl der in der Schweiz gebrauchten Instrumente sollte auf ein Minimum beschränkt bleiben, um Prozesse standardisieren zu können, Vergleiche unter den Notfallstationen zu ermöglichen (Benchmarking), Weiterbildung zu standardisieren, Mobilität von Pflegenden und Ärzten zu erleichtern und um schliesslich auch Forschung und Weiterentwicklungen mittels der gewählten Instrumente zu ermöglichen.
Unter den gemäss Literatur validierten Triageinstrumenten haben der Emergency Severity Index (ESI) und die Echelle Suisse de Tri (EST) die beste Validität und das geringste Risiko bezüglich Unter- und Übertriage.

Derzeit wird die EST in den zwei Westschweizer Universitätszentren wie auch in verschiedenen Regional- und Kantonsspitälern der Westschweiz und im Tessin verwendet. Der ESI ist hingegen in verschiedenen Universitäts- und Regionalspitälern der Deutschschweiz in Gebrauch. Beide Instrumente werden somit mit guter Erfahrung in verschiedenen wichtigen medizinischen Zentren eingesetzt.

Trotz der Vorteile, die eine totale Vereinheitlichung der Triageinstrumente in der Schweiz brächte, scheint es derzeit unmöglich, nur ein einziges Instrument schweizweit einzusetzen. Daher schlägt die SGNOR vor, sich wenigstens auf zwei Systeme zu beschränken und empfiehlt den Gebrauch des Emergency Severity Index oder der Echelle Suisse de Tri.

\section{Literatur}

1 Australasian College for Emergency Medicine Policy Document. The australasian triage scale. Emergency Medicine (Fremantle, W.A.) 2002; 14:(3)335-6.

2 Fernandes C, Tanabe P, Gilboy N, Johnson L, McNair $\mathrm{R}$, Rosenau A et al. Five level triage: a report from the ACEP/ENA five level task force. Journal of Emergency Nursing. 2005;31(1):39-50.

3 Hugli O, Moujber M, Simon J, Geissbuhler A, Yersin B, Sarasin F et al. Analyse de la fiabilité et de la performance de deux échelles de tri à l'aide d'un simulateur informatique. Journal Européen des Urgences. 2008;21:A107.

4 Mackway-Jones K. Emergency triage. 2nd edition. Manchester Triage Group. BMJ Publishing Group. 2006.

5 Murray M, Bullard M, Grafstein E, for the CTAS and CEDIS National Working Groups. Revisions to the Canadian Emergency Department Triage and Acuity Scale implementation guidelines. Can J Emerg Med, 2004;6 (6):421-7.

6 Rutschmann OT, Hugli O, Geissbuhler A, Kossovsky M, Simon J, Sarasin FP. Standardization of vital signs measurement during the triage process improves triage reliability. Ann Emerg Med. 2008;52:167-168.

8 Rutschmann OT, Kossovsky MP, Geissbuhler A, Perneger TV, Vermeulen B, Simon J et al. Interactive triage simulator revealed important variability in both process and outcome of emergency triage. Journal of Clinical Epidemiology. 2006;59(6):615-21.

9 Sieber R. Triage Process in Swiss Emergency Departments. Ann Emerg Med. 2008; 51:531. 